\title{
Endometriosis cutánea primaria umbilical: \\ a propósito de un caso
}

\section{Umbilical Primary Cutaneous Endometriosis: \\ A Case Report}

\section{Endometriose cutânea primária umbilical: relato de caso}

Ivan Lozada-Martínez, Est. ${ }^{1 *}$

Gian Núñez-Rojas, MD²

María Bolaño-Romero, Est. ${ }^{1}$

José Salcedo-Hurtado, MD²

Christian Ospina-Pérez, MD²

Recibido: 14 de septiembre de 2020 - Accepted: 25 de octubre de 2021

Doi: https://doi.org/10.12804/revistas.urosario.edu.co/revsalud/a.9458

Para citar este artículo: Lozada-Martínez I, Núñez-Rojas G, Bolaño-Romero M, Salcedo-Hurtado J, Ospina-Pérez C. Endometriosis cutánea primaria umbilical: a propósito de un caso. Rev Cienc Salud. 2022;20(1):1-7. https://doi.org/10.12804/revistas.urosario.edu.co/ revsalud/a.9458

\section{Resumen}

Introducción: la endometriosis es una de las patologías ginecológicas más frecuentes, que se entiende como la implantación intrapélvica de tejido endometrial, sensible, en la gran mayoría de casos, al influjo hormonal. Extrauterinamente, se caracteriza por la presencia de glándulas y estroma endometrial en órganos o tejidos no ginecológicos. Sin embargo, la piel corresponde a uno de los sitios con menor frecuencia de implantación, ya sea de forma primaria o secundaria. Se calcula la incidencia de endometriosis cutánea en la zona umbilical en menos del 1\% de los casos. Presentación del caso: mujer de 36 años de edad, quien consulta con Cirugía General por un cuadro clínico de dos años de evolución, consistente en lesiones sobrelevantadas de la piel en la región umbilical, de color café oscuro, las cuales describe

1 Grupo Prometheus y Biomedicina Aplicada a las Ciencias Clínicas, Facultad de Medicina, Universidad de Cartagena (Cartagena, Colombia).

2 Servicio de Cirugía General, ESE Hospital Universitario del Caribe, Universidad de Cartagena (Cartagena, Colombia).

Ivan Lozada-Martínez, oRciD https://orcid.org/0000-0002-1960-7334

Gian Núñez-Rojas, oRciD https://orcid.org/0000-0001-7729-4973

María Bolaño-Romero, oRCID https://orcid.org/0000-0001-7043-6617

José Salcedo-Hurtado, ORCID https://orcid.org/0000-0003-0932-5963

Christian Ospina-Pérez, oRCID https://orcid.org/0000-0002-9572-6829

* Autor de correspondencia: ivandavidloma@gmail.com 
como dolorosas y sangrantes en el momento del ciclo menstrual. A pesar de haber sido resecadas en dos ocasiones, se encontró persistencia del cuadro. Discusión: la endometriosis cutánea primaria umbilical representa del 0.5 al $1.0 \%$ de todas las pacientes con endometriosis ectópica, y el ombligo es uno de los sitios menos comunes de implantación. Esta condición afecta alrededor del 5 al $15 \%$ de mujeres en edad fértil, y del 3 al $5 \%$ de mujeres en edad menopaúsica. La presentación clínica más común se basa en una masa palpable, sangrado umbilical y dolor regular o irregular abdominal. Al asociar la endometriosis extrapélvica con la presencia de endometriosis pélvica, solamente alrededor del $20 \%$ de las pacientes presentará esta última.

Palabras clave: endometriosis; piel; ombligo; cirugía general; informes de casos.

\section{Abstract}

Introduction: Endometriosis is one of the most frequent gynecological pathologies, understood as an intrapelvic implantation of endometrial tissue, sensitive to hormonal influence in most cases. At the extrauterine level, it is characterized by the presence of endometrial glands and stroma in the non-gynecological organs or tissues. However, the skin corresponds to one of the sites with the least frequency of implantation, either primary or secondary, with the incidence of cutaneous endometriosis being calculated at the umbilical level in $<1 \%$ of the cases. Case presentation: A 36-year-old female patient presented to the General Surgery department with a two-year history of dark brown, raised skin lesions in the umbilical region, which she described as painful, and bleeding at the time of the menstrual cycle. Despite having been resected twice, the condition still persisted. Discussion: Umbilical primary cutaneous endometriosis represents $0.5 \%-1.0 \%$ of all patients with ectopic endometriosis, with the navel being one of the least common implantation sites. This condition affects about 5\%-15\% of women of childbearing age, and 3\%-5\% women of menopausal age. The most common clinical presentation is based on the palpable mass, umbilical bleeding, regular or irregular abdominal pain. Associating extrapelvic endometriosis with the presence of pelvic endometriosis, only about $20 \%$ of the patients will have the latter.

Keywords: Endometriosis; skin; umbilicus; general surgery; case report.

\section{Resumo}

Introdução: a endometriose é uma das patologias ginecológicas mais frequentes, entendida como o implante intrapélvico de tecido endometrial, sensível na grande maioria dos casos à influência hormonal. No nível extrauterino, é caracterizada pela presença de glândulas e estroma endometriais em órgãos ou tecidos não ginecológicos. Porém, a pele corresponde a um dos locais com menor frequência de implantação, seja ela primária ou secundária, calculando-se a incidência de endometriose cutânea ao nível umbilical em menos de $1 \%$ dos casos. Apresentação do caso: paciente do sexo feminino, com 36 anos de idade, que consulta para Cirurgia Geral devido a quadro clínico de 2 anos de evolução, constituído por lesões cutâneas elevadas na região umbilical, de coloração marrom-escura, que descreve como dolorosas e com sangramento no momento do ciclo menstrual. Apesar de ter sido ressecado em 2 ocasiões, o quadro era persistente. Discussão: a endometriose cutânea primária umbilical representa 0.5\%-1.0\% de todas as pacientes com endometriose ectópica, sendo o umbigo um dos locais de implantação menos comuns. Essa condição afeta cerca de 5\% a 15\% das mulheres em idade reprodutiva e 3\% a 5\% das mulheres em menopausa. A apresentação clínica mais comum baseia-se em massa palpável, sangramento umbilical, dor abdominal regular ou irregular. Ao associar a endometriose extrapélvica à presença de endometriose pélvica, apenas cerca de $20 \%$ das pacientes a apresentarão.

Palavras-chave: endometriose; pele; umbigo; cirurgia geral; relato de casos. 


\section{Introducción}

T a endometriosis se ha definido como la presencia de tejido endometrial no neoplásico ـfuera del útero. Esta entidad es de cierta forma común y afecta usualmente los ovarios. A partir de ello, genera una clínica basada en dolor pélvico profundo, dispareunia y dismenorrea. La endometriosis cutánea es excepcional, y ocurre cuando las glándulas endometriales y el estroma residen en la piel (1). La endometriosis cutánea se divide en primaria y secundaria. No se conoce mucho acerca de la patogenia de la endometriosis cutánea primaria, pero se cree que la secundaria se genera posterior a una cirugía abdominal o pélvica (2). Se ha descrito como una enfermedad benigna; sin embargo, se han encontrado registros que sugieren conversión a malignidad, lo que obliga a estudiar sus características histopatológicas, a fin de adoptar conductas terapéuticas asertivas (3). Y dado que la condición es extraordinaria, y puede aparentar otras condiciones cutáneas, como queloide o dermatofibroma, el diagnóstico suele ser un reto, por lo que se hace necesario exponer y analizar este tipo de entidad (4).

\section{Caso clínico}

Elirugía General por un cuadro clínico de dos años de evolución, consistente en lesiones sobrelevantadas de la piel en la región umbilical, de color café oscuro, que describió como dolorosas y sangrantes en el momento del ciclo menstrual. Estas habían sido resecadas en dos ocasiones. Como antecedentes relevantes, la mujer refirió enfermedad de ArnoldChiari, que había requerido manejo quirúrgico durante su infancia.

Desde el punto de vista ginecológico, presentó como fórmula obstétrica dos gestaciones con partos vaginales. Además, refirió ciclos irregulares y que no estaba planificando a la valoración. No tenía diagnóstico de endometriosis pélvica previo, ni cirugías ginecológicas por laparoscopia o laparotomías.

Su menarquia fue a los 11 años y tenía ocasional dispareunia sin sangrado en la relación. Negó antecedentes familiares de importancia. En el examen físico se evidenciaron dos lesiones nodulares: una de $0.4 \mathrm{~cm} \times 0.3 \mathrm{~cm}$ y otra de $0.2 \mathrm{~cm} \times 0.2 \mathrm{~cm}$, sobreelevadas, regulares, de bordes bien definidos, de color violáceo, dolorosas a la palpación, sin sangrado o secreción; además, la presencia de un defecto herniario en la pared abdominal en la región umbilical, de aproximadamente $1 \mathrm{~cm}$ de diámetro mayor, reductible (figura 1). Según lo descrito, se recibió la remisión con una impresión diagnóstica de hernia umbilical. 


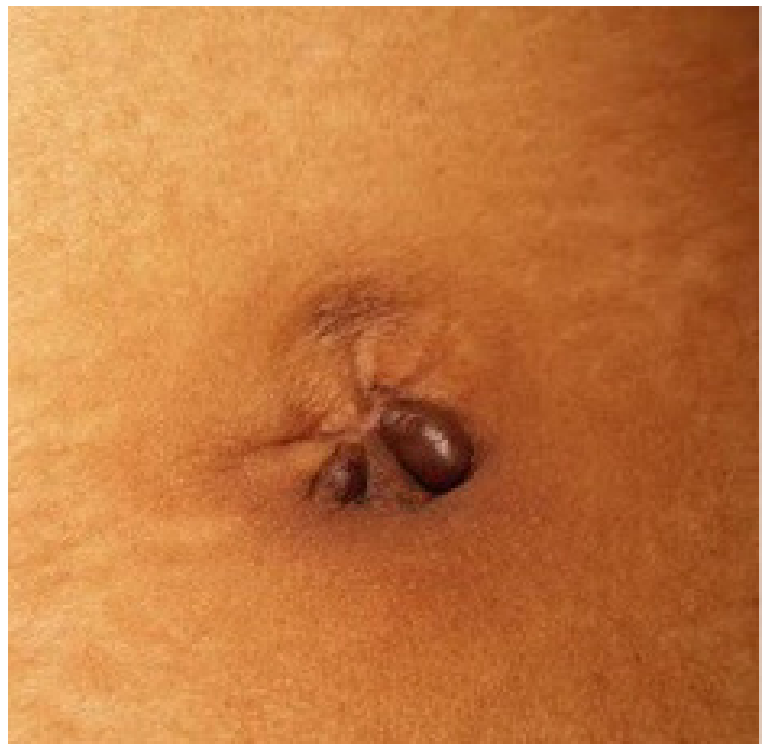

Figura 1. Lesiones periumbilicales dolorosas, violáceas, con bordes definidos, sangrantes con ciclos menstruales, que motivan la consulta

Posterior al análisis del caso, se planteó realizarle una cirugía para la exéresis de dichas lesiones y una herniorrafía umbilical sin colocación de malla, dado el tamaño del defecto. Dicho procedimiento se llevó a cabo sin complicaciones, mientras el tejido extirpado se envió a patología (figura 2). En el control por consulta, la paciente mostraba una buena evolución, estaba asintomática y sin dolor residual. Entre tanto, el resultado de patología informó tejido fibroconectivo y adiposo rodeado de glándulas con apariencia endometrial, revestida por epitelio cilíndrico sin atipias con alteración de su arquitectura y focos de hemorragia (focos endometriósicos), sin evidencia de malignidad. La herida quirúrgica tenía una evolución adecuada en el momento de la valoración.

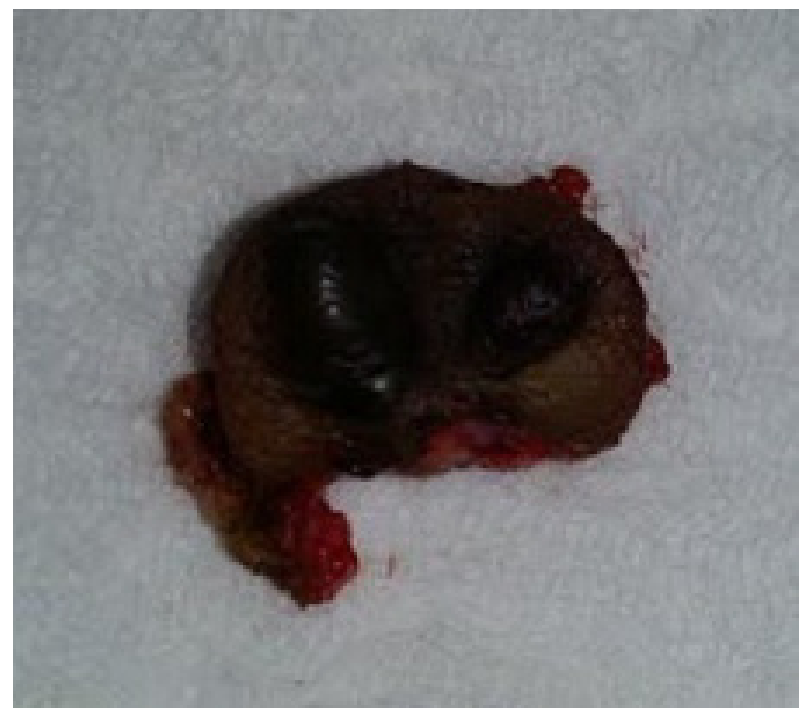

Figura 2. Tejido producto de la resección cutánea y de las lesiones periumbilicales 


\section{Discusión}

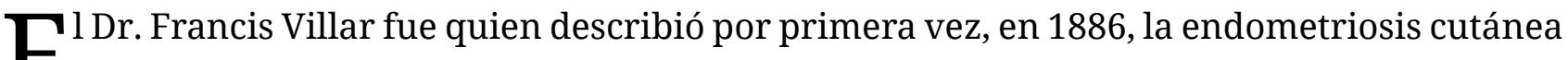
Elocalizada en el ombligo de una paciente, y nombró a esta lesión como el nódulo de Villar. La endometriosis cutánea primaria umbilical representa del $0.5 \%$ al $1.0 \%$ de todas los mujeres con endometriosis ectópica, y el ombligo es uno de los sitios menos comunes de implantación (5). Otras localizaciones reportadas han sido la parte superior de los órganos viscerales abdominales, la pared abdominal, el diafragma y la pleura, de las cuales se ha especulado su presencia como resultado de una diseminación peritoneal. Del mismo modo, se han encontrado lesiones en sitios más distantes, como el sistema nervioso central y el sistema nervioso periférico, posiblemente como resultado de una diseminación linfática o una hematológica (6).

Esta condición afecta alrededor del $5 \%$ al $15 \%$ de mujeres en edad fértil, y del $3 \%$ al $5 \%$ de mujeres en edad menopaúsica (7). En un metanálisis de Andres et al. (8) se evaluaron 88 estudios que habían incluido a 230 pacientes con endometriosis abdominal. De estas, 133 presentaban lesión en la ingle; 82, en la región umbilical; 13, en la pared abdominal, y 2, en el perineo. Todos los casos se clasificaron como lesiones primarias, es decir, estas mujeres no presentaban antecedentes de cirugías abdominales y perineales (8). La presentación clínica más común se basó en una masa palpable, sangrado umbilical y dolor regular o irregular abdominal.

Cuando los autores asociaron la endometriosis extrapélvica con la presencia de endometriosis pélvica en 134 pacientes, pero encontraron que solo el $18 \%$ la tenía. Es preciso resaltar que en los casos asociados las lesiones no se hallaban contiguas a la endometriosis pélvica (ingle) (8). Por lo anterior, podemos esperar que cuando la lesión esté distante a la región pélvica, no vamos a encontrar endometriosis pélvica; sin embargo, no debemos dejar pasar la oportunidad de hacer una evaluación completa, más aún si se trata de mujeres en edad fértil.

Nuestra paciente presentó la clínica común, con un dato clave, y es que refería el sangrado umbilical solo cuando se encontraba menstruando. La escisión quirúrgica es el tratamiento de elección para aquellas pacientes con nódulos solitarios ( $>5 \mathrm{~mm}-<5 \mathrm{~cm}$ ); mientras que en las lesiones difusas o grandes se prefiere el manejo con terapia hormonal o con anticonceptivos orales (9). El examen histopatológico de la pieza quirúrgica que evidencia la presencia de glándulas endometriales, asociado con focos hemorrágicos y con una clínica común, confirma el diagnóstico (10).

Como puntos de aprendizaje, tenemos que la endometriosis cutánea es una entidad de presentación rara, capaz de imitar otras entidades cutáneas, por lo que es necesario ser exhaustivo a la hora de realizar un diagnóstico diferencial. De ahí que se deba considerar la frecuencia de presentación en mujeres en edad fértil e, incluso, en mujeres en edad 
menopaúsica. Una buena historia clínica, con un examen físico asertivo, y la histopatología permiten un diagnóstico preciso, que permita una terapéutica eficaz.

\section{Contribución de los autores}

a concepción y diseño, la adquisición de los datos e información o análisis e interpreta-
ción de los datos: Ivan Lozada-Martínez, Gian Núñez-Rojas, María Bolaño-Romero, José Salcedo-Hurtado y Christian Ospina-Pérez.

Planeación del artículo o revisión de contenido intelectual importante: Ivan LozadaMartínez, Gian Núñez-Rojas, María Bolaño-Romero, José Salcedo-Hurtado y Christian Ospina-Pérez.

Aprobación final de la versión que se va a publicar: Ivan Lozada-Martínez, Gian NúñezRojas, María Bolaño-Romero, José Salcedo-Hurtado y Christian Ospina-Pérez.

\section{Conflicto de intereses}

Ninguno declarado.

\section{Referencias}

1. Raffi L, Suresh R, McCalmont T, Twigg A. Cutaneous endometriosis. Int J Women Dermatol. 2019;5(3):1-3. https://doi.org/10.1016/j.ijwd.2019.06.025

2. Loh SH, Lew BL, Sim WY. Primary cutaneous endometriosis of umbilicus. Ann Dermatol. 2017;29(5):621-5. https://doi.org/10.5021/ad.2017.29.5.621

3. Kajiyama H, Suzuki S, Yoshihara M, Tamauchi S, Yoshikawa N, Niimi K. Endometriosis and cancer. Free Radic Biol Med. 2019;133:186-92. https://doi.org/10.1016/j.freeradbiomed.2018.12.015

4. Cameron M, Westwell S, Subramanian A, Ramesar K, Howlett D. Postmenopausal cutaneous endometriosis: mimicking breast metastasis. Breast J. 2017;23(3):356-8. https:// doi.org/10.1111/tbj.12742

5. Victory R, Diamond MP, Johns DA. Villar's nodule: a case report and systematic literature review of endometriosis externa of the umbilicus. J Minim Invasive Gynecol. 2007;14:23-32. https://doi.org/10.1016/j.jmig.2006.07.014

6. Matalliotakis M, Goulielmos GN, Kalogiannidis I, Koumantakis G, Matalliotakis I, Arici A. Extra pelvic endometriosis: retrospective analysis on 200 cases in two different 
countries. Eur J Obstet Gynecol Reprod Biol. 2017;217:34-7. https://doi.org/10.1016/j. ejogrb.2017.08.019

7. Lopez A, Sanchez MI, Martinez JP, Ortiz S, Bernal CM, Remezal M. Cutaneous endometriosis: presentation of 33 cases and literature review. Eur J Obstet Gynecol Reprod Biol. 2018;221:58-63. https://doi.org/10.1016/j.ejogrb.2017.11.024

8. Andres MP, Arcoverde FV, Souza CC, Fernandes LF, Abrão MS, Kho RM. Extrapelvic endometriosis: a systematic review. J Minim Invasive Gynecol. 2019;:S1553-4650(19):31256-7. https://doi.org/10.1016/j.jmig.2019.10.004

9. Pandey N, Qayoom S, Sagar M, Goel MM. A rare case report of cutaneous endometriosis over arm diagnosed by fine needle aspiration cytology. Diagn Cytopathol. 2019;47(2):144-6. https://doi.org/10.1002/dc.23991

10. Pellegrin AE. Cutaneous decidualized endometriosis: a pseudomalignancy. Am J Dermatol. 1982;4:171-174. https://doi.org/10.1097/00000372-198204000-00012 